\title{
Exact and Heuristic Algorithms for 2-Layer Straightline Crossing Minimization
}

\author{
Michael Jünger ${ }^{1 *}$ and Petra Mutzel ${ }^{2 *}$ \\ 1 Institut für Informatik, Universität zu Köln, Pohligstr. 1, D-50969 Köln, Germany \\ 2 Max-Planck-Institut für Informatik, Im Stadtwald, D-66123 Saarbrücken, Germany
}

\begin{abstract}
We present algorithms for the two layer straightline crossing minimization problem that are able to compute exact optima. Our computational results lead us to the conclusion that there is no need for heuristics if one layer is fixed, even though the problem is NP-hard, and that for the general problem with two variable layers, true optima can be computed for sparse instances in which the smaller layer contains up to 15 nodes. For bigger instances, the iterated barycenter method turns out to be the method of choice among several popular heuristics whose performance we could assess by comparing the results to optimum solutions.
\end{abstract}

\section{Introduction}

Two layer straightline crossing minimization is receiving a lot of attention in automatic graph drawing. The problem consists of aligning the two shores $V_{1}$ and $V_{2}$ of a bipartite graph $G=\left(V_{1}, V_{2}, E\right)$ on two parallel straight lines (layers) such that the number of crossings between the edges in $E$ is minimized when the edges are drawn as straight lines connecting the endnodes. There appears to be a general agreement that good solutions for this problem contribute to better readability of diagrams representing hierarchical organizations on two or more layers.

Let $n_{1}=\left|V_{1}\right|, n_{2}=\left|V_{2}\right|, m=|E|$, and let $N(v)=\{w \in V \mid e=\{v, w\} \in E\}$ denote the set of neighbors of $v \in V=V_{1} \cup V_{2}$ in $G$. Any solution is obviously completely specified by a permutation $\pi_{1}$ of $V_{1}$ and a permutation $\pi_{2}$ of $V_{2}$. For $k=1,2$ let $\delta_{i j}^{k}=1$ if $\pi_{k}(i)<\pi_{k}(j)$ and 0 otherwise. Thus $\pi_{k}(k=1,2)$ is uniquely characterized by the vector $\delta^{k} \in\{0,1\}\left(\begin{array}{c}n_{h} \\ 2\end{array}\right)$. Given $\pi_{1}$ and $\pi_{2}$, the number of crossings is

$$
\begin{aligned}
C\left(\pi_{1}, \pi_{2}\right)=C\left(\delta^{1}, \delta^{2}\right) & =\sum_{i=1}^{n_{2}-1} \sum_{j=i+1}^{n_{2}} \sum_{k \in N(i)} \sum_{l \in N(j)} \delta_{k l}^{1} \cdot \delta_{j i}^{2}+\delta_{l k}^{1} \cdot \delta_{i j}^{2} \\
& =\sum_{k=1}^{n_{1}-1} \sum_{l=k+1}^{n_{1}} \sum_{i \in N(k)} \sum_{j \in N(l)} \delta_{k l}^{1} \cdot \delta_{j i}^{2}+\delta_{l k}^{1} \cdot \delta_{i j}^{2} .
\end{aligned}
$$

* Partially supported by DFG-Grant Ju204/7-1, Forschungsschwerpunkt “Effiziente Algorithmen für diskrete Probleme und ihre Anwendungen" 
It has been proven in [GJ83] that the two layer straightline crossing minimization problem is NP-hard, even if the permutation on one layer is fixed [EW94]. Therefore, a lot of effort went into the design of efficient heuristics, for the version in which one permutation is fixed as well as for the general case. Eades and Kelly [EK86] observe that the computation of true optima would be desirable in order to assess the performance of various heuristics, however, [EK86] believe that the NP-hardness of the problem renders such an experimental evaluation impractical.

In this paper, we would like to demonstrate that, if one permutation is fixed, it is indeed possible to compute the exact minima in surprisingly short computation times. In section 2, we outline our algorithm which transforms the problem to a linear ordering problem that is subsequently solved via the branch and cut method. In section 3 , we give computational results that allow us to assess the performance of several popular heuristics accurately.

Assume the permutation $\pi_{1}$ of $V_{1}$ is fixed. For each pair of nodes $i, j \in V_{2}$, $i \neq j$, we define $c_{i j}$ to be the number of crossings between edges incident with $i$ and edges incident with $j$ if $\pi_{2}$ is such that $\pi_{2}(i)<\pi_{2}(j)$. Then

$$
L=\sum_{i=1}^{n_{2}-1} \sum_{j=i+1}^{n_{2}} \min \left\{c_{i j}, c_{j i}\right\}
$$

is a trivial lower bound on the number of crossings. One observation in our experiments was that this trivial lower bound is surprisingly good. In section 4, we utilized this fact and the branch and cut algorithm of section 2 for the design and implementation of a program that solves the general two layer straightline crossing minimization problem to optimality.

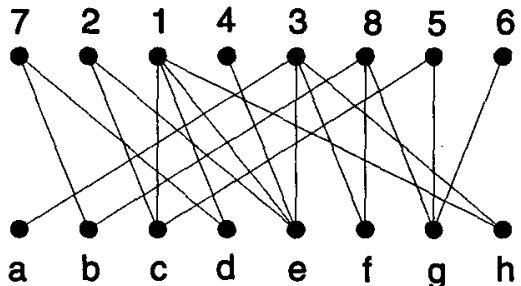

(a)

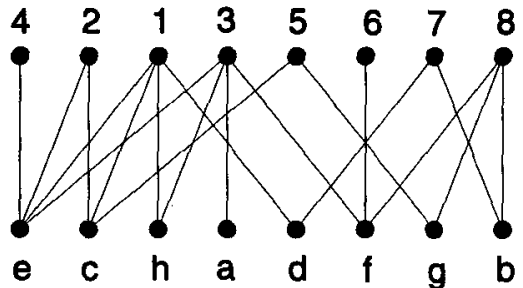

(b)

Fig. 1.

Figure 1 demonstrates that the number of crossings can indeed be considerably less if both layers can be freely permuted. The left drawing was given in [STT81] with fixed lower layer, [STT81] obtained the shown drawing with 48 crossings that we could show to be optimum. The right drawing is the optimum when both layers can be freely permuted. It has only 19 crossings.

As was to be expected, two sided crossing minimization can be done only for small instances. For large instances, we adopt the common method that consists of fixing the first layer, "optimizing" the second, fixing the found permutation 
of the second, "optimizing" the first, etc., back and forth, until the crossing number is not reduced anymore. We follow this iterative approach both using the heuristics of section 3 as well as the exact algorithm. The results are somewhat surprising, e.g., using the barycenter heuristic rather than exact one-sided crossing minimization yields slightly better results.

\section{Branch and Cut for One Sided Crossing Minimization}

The one sided straightline crossing minimization problem consists of fixing a permutation $\pi_{1}$ of $V_{1}$ and finding a permutation $\pi_{2}$ of $V_{2}$ such that the number of straightline crossings

$$
C\left(\pi_{2}\right)=C\left(\delta^{2}\right)=\sum_{i=1}^{n_{2}-1} \sum_{j=i+1}^{n_{2}} \sum_{k \in N(i)} \sum_{l \in N(j)} \delta_{k l}^{1} \cdot \delta_{j i}^{2}+\delta_{l k}^{1} \cdot \delta_{i j}^{2}
$$

is minimized. Let

$$
c_{i j}=\sum_{k \in N(i)} \sum_{l \in N(j)} \delta_{l k}^{1}
$$

denote the number of crossings among the edges adjacent to $i$ and $j$ if $\pi_{2}(i)<$ $\pi_{2}(j)$. Then

$$
\begin{aligned}
C\left(\pi_{2}\right)=C\left(\delta^{2}\right) & =\sum_{i=1}^{n_{2}-1} \sum_{j=i+1}^{n_{2}} c_{i j} \delta_{i j}^{2}+c_{j i}\left(1-\delta_{i j}^{2}\right) \\
& =\sum_{i=1}^{n_{2}-1} \sum_{j=i+1}^{n_{2}}\left(c_{i j}-c_{j i}\right) \delta_{i j}^{2}+\sum_{i=1}^{n_{2}-1} \sum_{j=i+1}^{n_{2}} c_{j i} .
\end{aligned}
$$

For $n=n_{2}, x_{i j}=\delta_{i j}^{2}$ and $a_{i j}=c_{i j}-c_{j i}$ we solve the linear ordering problem

$$
\text { (LO) minimize } \begin{aligned}
& \sum_{i=1}^{n_{2}-1} \sum_{j=i+1}^{n_{2}} a_{i j} x_{i j} \\
& 0 \leq x_{i j}+x_{j k}-x_{i k} \leq 1 \quad \text { for } 1 \leq i<j<k \leq n \\
& 0 \leq x_{i j} \leq 1 \text { for } 1 \leq i<j \leq n \\
& x_{i j} \in\{0,1\} \text { for } 1 \leq i<j \leq n .
\end{aligned}
$$

If $z$ is the optimum value of (LO), $z+\sum_{i=1}^{n_{2}-1} \sum_{j=i+1}^{n_{2}} c_{j i}$ is the minimum number of crossings.

The constraints of (LO) guarantee that the solutions correspond indeed precisely to all permutations $\pi_{2}$ of $V_{2}$. Furthermore, it can be shown that the "3-cycle constraints" are necessary in any minimal description of the feasible solutions by linear inequalities, if the integrality conditions are dropped. The NP-hardness of the problem makes it unlikely that such a complete linear description can be found and exploited algorithmically. Further classes of inequalities with a 
number of members exponential in $n$ that must be present in a complete linear description of the feasible set, are known, and some of them can be exploited algorithmically. For the details see [GJR85].

When the integrality conditions in (LO) are dropped, only $2\left(\begin{array}{c}n \\ 2\end{array}\right)$ hypercube inequalities and $2\left(\begin{array}{l}n \\ 3\end{array}\right)$ 3-cycle inequalities are left that define a relaxation of (LO) which has been proven very useful in practical applications. In [GJR84a] a branch and cut algorithm for (LO) is proposed that solves this relaxation with a cutting plane approach, since writing down all 3-cycle inequalities, even though taking only polynomial space, and solving the corresponding linear program, is not practical for space reasons. Rather the algorithm starts with the hypercube constraints that are handled implicitly by the LP-solver, and iteratively adds violated 3-cycle constraints and deletes nonbinding 3-cycle constraints after an LP has been solved, until the relaxation is solved. If the optimum solution is integral, the algorithm stops, otherwise it is applied recursively to two subproblems in one of which a fractional $x_{i j}$ is set to 1 and in the other set to 0 . In [GJR84b] such a branch and cut approach could be used to find optimum linear orderings with $n$ up to 60 in an application involving input-output matrices that are used in economic analysis. For the many details and the inclusion of further useful inequalities in the cutting plane part, see [GJR84a].

A new implementation of the algorithm is used in our computational experiments. It is written in C and uses the [CPLEX] software for solving the linear programming relaxations coming up in the course of the computation.

\section{One Sided Crossing Minimization}

The fact that we are able to compute optimum solutions allows us to assess the quality of various popular heuristics for one-sided two layer straightline crossing minimization experimentally. Our computational comparison includes the following heuristics: the barycenter heuristic by [STT81], the median heuristic by [EW94], the stochastic heuristic by [D94], the greedy-insert heuristic by [EK86], the greedy-switch heuristic by [EK86], and the split heuristic by [EK86].

In order to gain confidence in the correctness of our implementations, we repeated the computational tests in [EK86]. We could reproduce their results accurately. There are no published computational results for the stochastic heuristic, but a personal communication with the author [D95] confirms the correctness of our implementation.

All subsequent tables have the following columns:

- $n_{i}$ : Number of nodes on layer $i$ for $i=1,2$

- $m$ : Number of edges

- Low: The trivial lower bound for the number of crossings

- Min: The minimum number of crossings (computed by the branch and cut algorithm)

- Bary: The number of crossings found by the barycenter heuristic

- Median: The number of crossings found by the median heuristic

- Stoch: The number of crossings found by the stochastic heuristic 
- Gre-ins: The number of crossings found by the greedy-insert heuristic

- Gre-swi: The number of crossings found by the greedy-switch heuristic

- Split: The number of crossings found by the split heuristic

For each type of graph, three numbers are given: the average number of crossings taken over all sampled instances of this type, the relative size of this number in percentage of the minimum number of crossings, and the average running time in seconds on a SUN Sparcstation 10. All samples are generated by the program random_bigraph of the Stanford GraphBase by Knuth [K93]. The generators are hardware independent and are available from the authors so that exactly the same experiments can be run by anyone who is interested.

In Table 1, we give the results for " $20+20$-graph", i.e., bipartite graphs with 20 nodes on each layer and various fixed numbers of edges chosen uniformly and independently from the set of all possible edges. Each average is taken over 100 samples. The most surprising fact is perhaps that the exact computation by the branch and cut algorithm is faster than many of the heuristics. Only the barycenter and the median heuristic are between two to four times faster than the exact algorithm. Furthermore, the table indicates, less surprisingly, that dense instances are not very interesting. The data is visualized in Figure 2.

Table 1. Results for 100 instances on $20+20$ nodes with increasing density

\begin{tabular}{|c|c|c|c|c|c|c|c|c|c|}
\hline$n_{i}$ & $m$ & Low & Min & Bary & Median & Stoch & Gre-ins & Gre-swi & Split \\
\hline 20 & 40 & $\begin{array}{r}180.35 \\
99.78\end{array}$ & $\begin{array}{r}180.75 \\
100.00 \\
0.02\end{array}$ & $\begin{array}{r}185.34 \\
102.54 \\
0.01\end{array}$ & $\begin{array}{r}206.27 \\
114.12 \\
0.01\end{array}$ & $\begin{array}{r}185.44 \\
102.60 \\
0.05\end{array}$ & $\begin{array}{r}248.37 \\
137.41 \\
0.02\end{array}$ & $\begin{array}{r}275.99 \\
152.69 \\
0.04\end{array}$ & $\begin{array}{r}183.39 \\
101.46 \\
0.08\end{array}$ \\
\hline 20 & 80 & $\begin{array}{r}957.62 \\
99.83\end{array}$ & $\begin{array}{r}959.23 \\
100.00 \\
0.03\end{array}$ & $\begin{array}{r}968.80 \\
101.00 \\
0.01\end{array}$ & $\begin{array}{r}1051.14 \\
109.58 \\
0.01\end{array}$ & $\begin{array}{r}970.01 \\
101.12 \\
0.06\end{array}$ & $\begin{array}{r}1175.11 \\
122.51 \\
0.05\end{array}$ & $\begin{array}{r}1044.14 \\
108.85 \\
0.10\end{array}$ & $\begin{array}{r}964.35 \\
100.53 \\
0.11\end{array}$ \\
\hline 20 & 120 & $\begin{array}{r}2420.14 \\
99.91\end{array}$ & $\begin{array}{r}2422.32 \\
100.00 \\
0.03 \\
\end{array}$ & $\begin{array}{r}2433.53 \\
100.46 \\
0.01 \\
\end{array}$ & $\begin{array}{r}2564.82 \\
105.88 \\
0.01 \\
\end{array}$ & $\begin{array}{r}2437.39 \\
100.62 \\
0.07 \\
\end{array}$ & $\begin{array}{r}2763.72 \\
114.09 \\
0.10 \\
\end{array}$ & $\begin{array}{r}2460.94 \\
101.59 \\
0.16 \\
\end{array}$ & $\begin{array}{r}2428.23 \\
100.24 \\
0.16 \\
\end{array}$ \\
\hline 20 & 160 & $\begin{array}{r}4625.79 \\
99.96\end{array}$ & $\begin{array}{r}4627.72 \\
100.00 \\
0.04 \\
\end{array}$ & $\begin{array}{r}4638.24 \\
100.23 \\
0.01 \\
\end{array}$ & $\begin{array}{r}4825.06 \\
104.26 \\
0.02 \\
\end{array}$ & $\begin{array}{r}4644.35 \\
100.36 \\
0.08 \\
\end{array}$ & $\begin{array}{r}5098.27 \\
110.17 \\
0.17\end{array}$ & $\begin{array}{r}4644.10 \\
100.35 \\
0.23\end{array}$ & $\begin{array}{r}4632.17 \\
100.10 \\
0.23\end{array}$ \\
\hline 20 & 200 & $\begin{array}{r}7560.42 \\
99.98\end{array}$ & $\begin{array}{r}7561.88 \\
100.00 \\
0.05 \\
\end{array}$ & $\begin{array}{r}7571.08 \\
100.12 \\
0.02 \\
\end{array}$ & $\begin{array}{r}7817.99 \\
103.39 \\
0.02 \\
\end{array}$ & $\begin{array}{r}7582.47 \\
100.27 \\
0.09\end{array}$ & $\begin{array}{r}8157.86 \\
107.88 \\
0.24 \\
\end{array}$ & $\begin{array}{r}7572.24 \\
100.14 \\
0.31 \\
\end{array}$ & $\begin{array}{r}7566.79 \\
100.07 \\
0.31 \\
\end{array}$ \\
\hline 20 & 240 & $\begin{array}{r}11314.37 \\
99.99\end{array}$ & $\begin{array}{r}11315.55 \\
100.00 \\
0.07 \\
\end{array}$ & $\begin{array}{r}1323.26 \\
100.07 \\
0.02 \\
\end{array}$ & $\begin{array}{r}11625.54 \\
102.74 \\
0.03 \\
\end{array}$ & $\begin{array}{r}11338.06 \\
100.20 \\
0.09 \\
\end{array}$ & $\begin{array}{r}12033.34 \\
106.34 \\
0.34 \\
\end{array}$ & $\begin{array}{r}1321.10 \\
100.05 \\
0.42 \\
\end{array}$ & $\begin{array}{r}11318.68 \\
100.03 \\
0.41 \\
\end{array}$ \\
\hline 20 & 280 & $\begin{array}{r}15859.70 \\
99.99\end{array}$ & $\begin{array}{r}15860.35 \\
100.00 \\
0.09 \\
\end{array}$ & $\begin{array}{r}15865.69 \\
100.03 \\
0.03 \\
\end{array}$ & $\begin{array}{r}16225.57 \\
102.30 \\
0.03 \\
\end{array}$ & $\begin{array}{r}15883.69 \\
100.15 \\
0.10\end{array}$ & $\begin{array}{r}16667.12 \\
105.09 \\
0.45 \\
\end{array}$ & $\begin{array}{r}15863.66 \\
100.02 \\
0.52\end{array}$ & $\begin{array}{r}15861.76 \\
100.01 \\
0.53\end{array}$ \\
\hline 20 & 320 & $\begin{array}{r}21290.56 \\
99.99\end{array}$ & $\begin{array}{r}21290.76 \\
100.00 \\
0.11 \\
\end{array}$ & $\begin{array}{r}21294.12 \\
100.02 \\
0.03 \\
\end{array}$ & $\begin{array}{r}21727.43 \\
102.05 \\
0.04 \\
\end{array}$ & $\begin{array}{r}21313.78 \\
100.12 \\
0.11 \\
\end{array}$ & $\begin{array}{r}2116.56 \\
103.88 \\
0.59 \\
\end{array}$ & $\begin{array}{r}21292.93 \\
100.01 \\
0.65 \\
\end{array}$ & $\begin{array}{r}21291.56 \\
100.00 \\
0.66 \\
\end{array}$ \\
\hline 20 & 360 & $\begin{array}{r}27751.63 \\
100.00\end{array}$ & $\begin{array}{r}27751.69 \\
100.00 \\
0.14\end{array}$ & $\begin{array}{r}27752.99 \\
100.01 \\
0.04 \\
\end{array}$ & $\begin{array}{r}28257.47 \\
101.82 \\
0.04\end{array}$ & $\begin{array}{r}27768.41 \\
100.06 \\
0.12 \\
\end{array}$ & $\begin{array}{r}28459.57 \\
102.55 \\
0.74 \\
\end{array}$ & $\begin{array}{r}27752.01 \\
100.00 \\
0.81\end{array}$ & $\begin{array}{r}27751.84 \\
100.00 \\
0.80 \\
\end{array}$ \\
\hline
\end{tabular}

In Table 2, we concentrate on sparse instances in which, on the average, every node has two adjacent edges. We believe that such instances are among the most interesting in practical applications. It turns out that the barycenter, the 

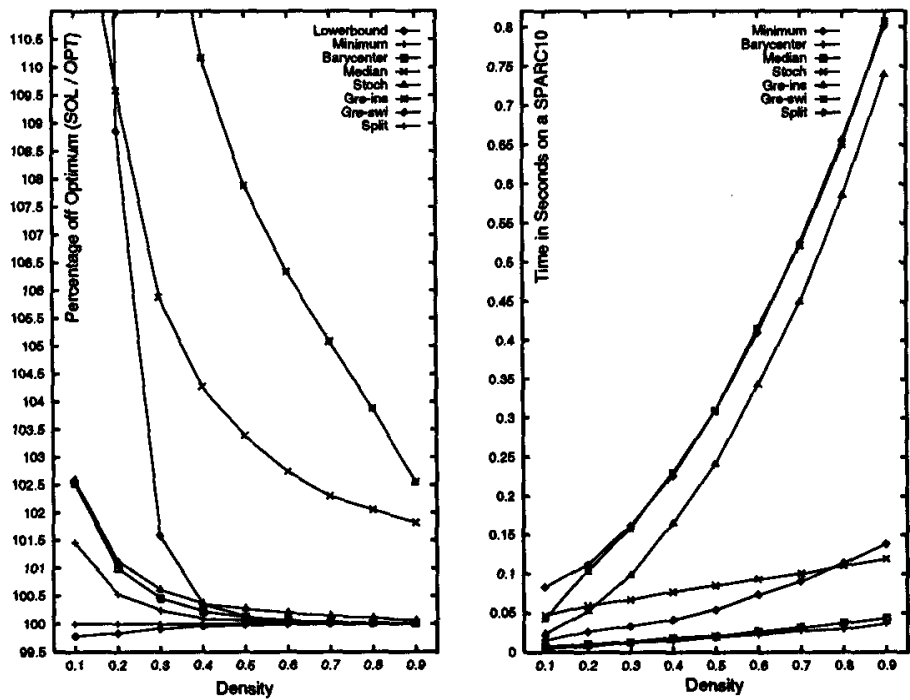

Fig. 2. Results for 100 instances on $20+20$ nodes with increasing density

Table 2. Results for 10 instances of sparse graphs with increasing size

\begin{tabular}{|r|r|r|r|r|r|r|r|r|r|}
\hline$n_{i}$ & $m$ & Low & Min & Bary & Median & Stoch & Gre-ins & Gre-swi & Split \\
\hline 10 & 20 & 37.90 & 38.00 & 38.90 & 45.40 & 38.70 & 46.40 & 50.90 & 38.50 \\
& & 99.74 & 100.00 & 102.37 & 119.47 & 101.84 & 122.11 & 133.94 & 101.32 \\
& & & 0.00 & 0.00 & 0.00 & 0.01 & 0.00 & 0.01 & 0.02 \\
\hline 20 & 40 & 171.70 & 171.90 & 175.70 & 193.70 & 174.90 & 240.80 & 293.60 & 174.70 \\
& & 99.88 & 100.00 & 102.21 & 112.68 & 101.74 & 140.08 & 170.80 & 101.63 \\
& & & 0.01 & 0.01 & 0.01 & 0.05 & 0.02 & 0.05 & 0.09 \\
\hline 30 & 60 & 436.60 & 438.30 & 451.90 & 491.10 & 451.30 & 602.30 & 692.40 & 445.60 \\
& & 99.61 & 100.00 & 103.10 & 112.05 & 102.87 & 137.42 & 157.97 & 101.67 \\
& & & 0.11 & 0.01 & 0.01 & 0.13 & 0.05 & 0.11 & 0.25 \\
\hline 40 & 80 & 761.50 & 765.70 & 785.60 & 856.60 & 782.70 & 1105.00 & 1367.50 & 783.20 \\
& & 99.45 & 100.00 & 102.60 & 111.87 & 102.22 & 144.31 & 178.60 & 102.29 \\
& & & 0.30 & 0.01 & 0.02 & 0.28 & 0.08 & 0.22 & 0.57 \\
\hline 50 & 100 & 1247.30 & 1252.20 & 1279.90 & 1389.50 & 1273.20 & 1770.60 & 2200.50 & 1277.80 \\
& & 99.61 & 100.00 & 102.21 & 110.97 & 101.68 & 141.40 & 175.73 & 102.04 \\
& & & 0.68 & 0.02 & 0.03 & 0.50 & 0.13 & 0.32 & 1.00 \\
\hline 60 & 120 & 1683.10 & 1687.60 & 1738.30 & 1890.90 & 1720.20 & 2453.10 & 2994.50 & 1736.10 \\
& & 99.73 & 100.00 & 103.00 & 112.05 & 101.93 & 145.36 & 177.44 & 102.87 \\
& & & 1.09 & 0.03 & 0.04 & 0.83 & 0.18 & 0.61 & 1.67 \\
\hline 70 & 140 & 2465.00 & 2479.00 & 2541.30 & 2730.00 & 2522.50 & 3592.20 & 4498.80 & 2549.20 \\
& & 99.44 & 100.00 & 102.51 & 110.13 & 101.76 & 144.91 & 181.48 & 102.83 \\
& & & 4.46 & 0.04 & 0.04 & 1.28 & 0.26 & 0.73 & 2.82 \\
\hline 80 & 160 & 3153.90 & 3172.10 & 3254.60 & 3521.60 & 3232.90 & 4583.10 & 5885.70 & 3240.60 \\
& & 99.43 & 100.00 & 102.60 & 111.02 & 101.92 & 144.48 & 185.55 & 102.16 \\
& & & 6.42 & 0.05 & 0.06 & 1.85 & 0.33 & 0.99 & 4.11 \\
\hline 80 & 180 & 4104.00 & 4132.80 & 4233.70 & 4566.80 & 4206.80 & 5843.70 & 7331.30 & 4293.90 \\
& & 99.30 & 100.00 & 102.44 & 110.50 & 101.79 & 141.40 & 177.39 & 103.90 \\
& & & 25.13 & 0.05 & 0.06 & 2.66 & 0.41 & 1.32 & 5.84 \\
\hline 100 & 200 & 5127.40 & 5162.70 & 5287.50 & 5728.80 & 5247.60 & 7469.90 & 9407.50 & 5333.50 \\
& & 99.32 & 100.00 & 102.42 & 110.97 & 101.64 & 144.69 & 182.22 & 103.31 \\
& & & 435.51 & 0.06 & 0.08 & 3.35 & 0.49 & 1.45 & 7.56 \\
\hline
\end{tabular}



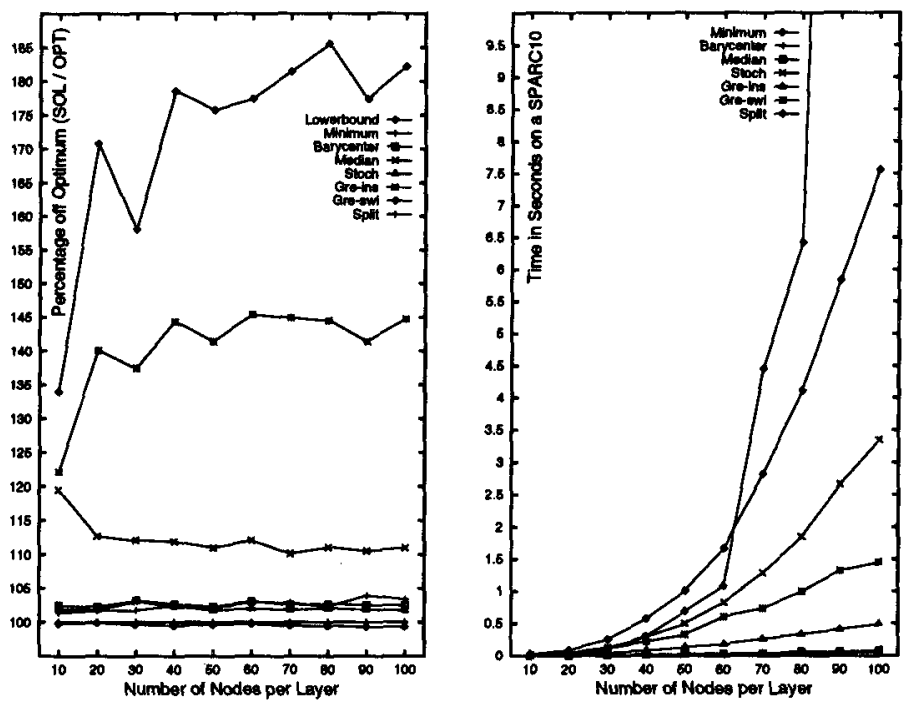

Fig. 3. Results for 10 instances of sparse graphs with increasing size

stochastic and the split heuristic perform very well in terms of quality, however, the split heuristic takes roughly the same time as the branch and cut computation up to size $80+80$, whereas the barycenter heuristic obtains results of similar quality as split, but much faster (see Figure 3).

In Table 3, we repeat an experiment by Dresbach [D94] for instances defined by Warfield [W77] as follows: For $k=3,4,5,6,7,8$ we let $n_{1}=k, n_{2}=2^{k}-1$, and the adjacency matrix of the bipartite graph is a $n_{1} \times n_{2}$ matrix whose rows are labelled $1,2, \ldots, k$, whose columns are labelled $1,2, \ldots, 2^{k}-1$, and column $j$ contains $j$ in $k$-digit binary notation. Layer 1 is fixed and layer 2 is "optimized". Again, it turns out that barycenter is the fastest method with excellent quality solutions. The results of the stochastic heuristic, the barycenter and the split heuristic are very close to the optimum solution. Up to size $7+127$, the branch and cut algorithm needs only moderate computation time, for the instance $8+255$ it is not competitive in terms of time, but we found it surprising that such a big linear ordering instance with $n=255$ could be solved at all. The branch and cut algorithm was the only method that found the true optima for $k \geq 6$, whereas for $3 \leq k \leq 5$, the fact that the optimum value equals the value of the trivial lower bound seems to indicate that these instances are not hard.

\section{Two Sided Crossing Minimization}

The trivial lower bound on the number of crossings that turned out to be excellent in our previous experiments, can obviously be adapted to partial orderings rather than complete orderings (permutations) on one of the layers. This encouraged us to devise a simple branch and bound algorithm for the general two layer straightline crossing minimization problem in which both $\pi_{1}$ and $\pi_{2}$ must 
Table 3. Results for Dresbach instances

\begin{tabular}{|l|r|r|r|r|r|r|r|r|r|r|}
\hline$n_{1}$ & $n_{2}$ & $m$ & Low & Min & Bary & Median & Stoch & Gre-ins & Gre-swi & Split \\
\hline 3 & 7 & 12 & 8 & 8 & 8 & 13 & 8 & 11 & 8 & 8 \\
& & & & 100.00 & 100.00 & 162.50 & 100.00 & 137.50 & 100.00 & 100.00 \\
& & & & 0.00 & 0.00 & 0.00 & 0.02 & 0.00 & 0.00 & 0.02 \\
\hline 4 & 15 & 32 & 95 & 95 & 95 & 127 & 95 & 122 & 98 & 95 \\
& & & & 100.00 & 100.00 & 133.68 & 100.00 & 128.42 & 103.16 & 100.00 \\
& & & & 0.00 & 0.00 & 0.00 & 0.03 & 0.02 & 0.05 & 0.07 \\
\hline 5 & 31 & 80 & 756 & 756 & 758 & 922 & 756 & 934 & 804 & 760 \\
& & & & 100.00 & 100.27 & 121.96 & 100.00 & 123.55 & 106.35 & 100.53 \\
& & & & 0.03 & 0.00 & 0.03 & 0.18 & 0.08 & 0.40 & 0.43 \\
\hline 6 & 63 & 192 & 4998 & 5002 & 5015 & 5818 & 5004 & 6023 & 5523 & 5043 \\
& & & & 100.00 & 100.26 & 116.31 & 100.04 & 120.41 & 110.42 & 100.90 \\
& & & & 0.73 & 0.05 & 0.07 & 1.38 & 0.38 & 2.87 & 2.65 \\
\hline 7 & 127 & 448 & 29745 & 29778 & 29883 & 33641 & 29841 & 35152 & 34366 & 30086 \\
& & & & 100.00 & 100.35 & 112.97 & 100.21 & 118.05 & 115.41 & 101.03 \\
& & & & 20.50 & 0.17 & 0.20 & 9.02 & 1.98 & 20.20 & 24.30 \\
\hline 8 & 255 & 1024 & 165375 & 165602 & 166098 & 183342 & 165824 & 192633 & 202957 & 167546 \\
& & & 100.00 & 100.30 & 110.71 & 100.13 & 116.32 & 122.56 & 101.17 \\
& & & & 7200.00 & 0.95 & 1.08 & 67.90 & 7.33 & 147.00 & 189.00 \\
\hline
\end{tabular}

be determined. Namely, we enumerate all permutations $\pi_{1}$ (let without loss of generality $\left.\left|V_{1}\right| \leq\left|V_{2}\right|, V_{1}=\{1,2, \ldots, n\}\right)$ as follows: Initially all $v \in V_{1}$ are unfixed. At depth $l$ in a depth-first-search, $l-1$ nodes of $V_{1}$ are fixed in positions $1,2, \ldots, l-1$. Then the first unfixed node in the canonical ordering of $V_{1}$ is fixed at position $l$, and the trivial lower bound $L$ is computed for the resulting partial ordering. If $L$ is greater than the value of the best known solution, the next unfixed node in the canonical ordering of $V_{1}$ is fixed at position $l$, else we move to position $l+1$, if $l<n$, and otherwise $(l=n)$ we call the branch and cut algorithm to determine an optimum ordering of $V_{2}$ and update the best known solution, if necessary. Backtracking, i.e. moving from position $l$ to position $l-1$ occurs whenever the list of unfixed nodes at depth $l$ in the enumeration tree is exhausted. Before the enumeration is entered, a heuristic solution is determined in order to initialize the best known solution. A good initial solution makes the enumeration tree smaller.

In Table 4, we use this algorithm to determine optimum solutions for $10+10$ graphs with increasing edge densities, 100 samples for each type of graph. It turns out that with increasing density, the computation times increase rapidly for the minimum computation, whereas the heuristics are not very sensitive to density. All heuristics are iterated between the two layers until a local optimum is obtained, as outlined in the introduction, starting from the canonical ordering on $V_{1}$. An additional column labelled "LR-Opt" gives according results for the iterated minimum crossing computation by branch and cut, which is, remarkably, sometimes outperformed by the best iterated heuristics. For sparse instances, the minimum is much better than any of the heuristically found solutions. In Figure 4, we show an example of a $10+10$ graph with 20 edges. The first drawing was found by the LR-Opt heuristic and has 30 crossings, the second by the barycenter heuristic and contains 11 crossings and the third one is the optimum solution with only 4 crossings. 

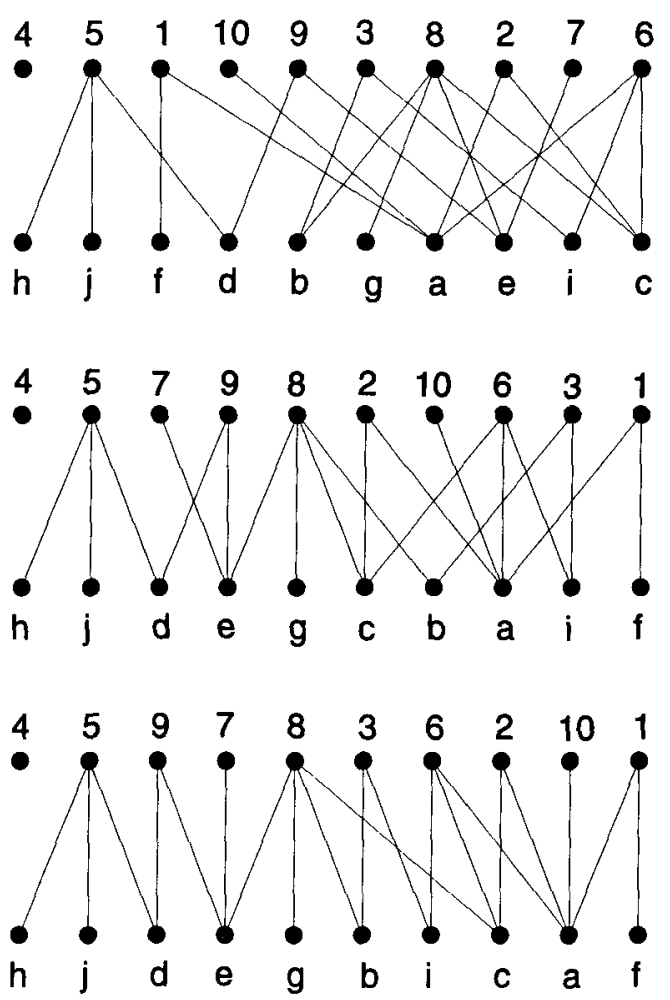

Fig. 4 .

Within one hour of computation time, we can find optimum solutions for $11+11$ instances with up to $80 \%$ density, $12+12$ with up to $50 \%$ density, $13+13$ with up to $30 \%$ density, $14+14,15+15,16+16$ with up to $10 \%$ density.

In Table 5, we repeat the same experiment with 10 starts from random orderings of the nodes in $V_{1}$. The results show that this way a considerable performance gain for all heuristics can be achieved. LR-Opt, Barycenter and Split obtain results of similar good quality.

Tables 6 and 7 deal with the more interesting sparse instances of bigger size for which we can not compute the optimum anymore, Table 6 with canonical start, Table 7 with 10 random starts. Summarizing, the barycenter method turns out to be the clear winner, both in terms of quality as well as in terms of computation time. 
Table 4. Results for 100 instances on $10+10$ nodes with increasing density

\begin{tabular}{|r|r|r|r|r|r|r|r|r|r|}
\hline$n_{i}$ & $m$ & Min & LR-Opt & Bary & Median & Stoch & Gre-ins & Gre-swi & Split \\
\hline 10 & 10 & 0.29 & 1.64 & 1.52 & 1.53 & 2.71 & 4.32 & 9.61 & 2.63 \\
& & 100.00 & 565.52 & 524.14 & 527.59 & 934.48 & 1489.66 & 3313.79 & 906.90 \\
& & 1.10 & 0.01 & 0.01 & 0.01 & 0.03 & 0.02 & 0.02 & 0.04 \\
\hline 10 & 20 & 11.62 & 19.99 & 18.78 & 24.08 & 26.96 & 38.85 & 34.81 & 23.25 \\
& & 100.00 & 172.03 & 161.62 & 207.23 & 232.01 & 334.34 & 299.57 & 200.09 \\
& & 3.89 & 0.02 & 0.01 & 0.01 & 0.06 & 0.04 & 0.03 & 0.07 \\
\hline 10 & 30 & 56.60 & 66.98 & 65.30 & 81.78 & 82.98 & 109.96 & 80.29 & 70.11 \\
& & 100.00 & 118.34 & 115.37 & 144.49 & 146.61 & 194.28 & 141.86 & 123.87 \\
& & 14.06 & 0.02 & 0.02 & 0.02 & 0.07 & 0.06 & 0.07 & 0.11 \\
\hline 10 & 40 & 146.89 & 157.91 & 157.70 & 189.55 & 182.77 & 225.26 & 165.65 & 160.20 \\
& & 100.00 & 107.50 & 107.36 & 129.04 & 124.43 & 153.35 & 112.77 & 109.06 \\
& & 43.02 & 0.03 & 0.02 & 0.02 & 0.08 & 0.10 & 0.11 & 0.15 \\
\hline 10 & 50 & 276.78 & 287.32 & 288.15 & 333.25 & 320.21 & 387.87 & 296.38 & 290.79 \\
& & 100.00 & 103.81 & 104.11 & 120.40 & 115.69 & 140.14 & 107.08 & 105.06 \\
& & 91.58 & 0.04 & 0.03 & 0.02 & 0.09 & 0.13 & 0.15 & 0.21 \\
\hline 10 & 60 & 463.17 & 475.04 & 475.52 & 539.59 & 509.38 & 598.98 & 482.76 & 478.46 \\
& & 100.00 & 102.56 & 102.67 & 116.50 & 109.98 & 129.32 & 104.23 & 103.30 \\
& & 206.61 & 0.06 & 0.03 & 0.03 & 0.10 & 0.17 & 0.22 & 0.28 \\
\hline 10 & 70 & 698.35 & 709.91 & 710.88 & 782.33 & 747.20 & 854.61 & 715.73 & 712.73 \\
& & 100.00 & 101.66 & 101.79 & 112.03 & 107.00 & 122.38 & 102.49 & 102.06 \\
& & 379.12 & 0.07 & 0.04 & 0.03 & 0.11 & 0.22 & 0.29 & 0.35 \\
\hline 10 & 80 & 1008.38 & 1021.46 & 1021.44 & 1110.39 & 1051.66 & 1165.07 & 1025.84 & 1024.78 \\
& & 100.00 & 101.30 & 101.30 & 110.12 & 104.29 & 115.63 & 101.73 & 101.63 \\
& & 763.53 & 0.08 & 0.04 & 0.03 & 0.12 & 0.27 & 0.34 & 0.40 \\
\hline 10 & 90 & 1405.57 & 1420.68 & 1421.86 & 1524.18 & 1430.86 & 1516.62 & 1423.90 & 1421.72 \\
& & 100.00 & 101.08 & 101.16 & 108.44 & 101.80 & 107.90 & 101.30 & 101.15 \\
& & 1549.12 & 0.07 & 0.03 & 0.03 & 0.12 & 0.29 & 0.32 & 0.37 \\
\hline
\end{tabular}

\section{Conclusions}

The outcome of our computational experiments lead to the following conclusions.

(1) When one layer is fixed, the exact minimum crossing number can be efficiently computed in practice, so there is no real need for heuristics.

(2) In the general case, small sparse instances as they occur in applications can be solved to optimality if the smaller sized shore has up to about 15 vertices. For larger instances, the iterated barycenter method, started with a few random orderings of one layer, is clearly the method of choice among all tested methods.

\section{Acknowledgements}

We would like to thank Thomas Ziegler for implementing and running all heuristics in this paper except LR-Opt and Stefan Dresbach for helpful discussions concerning the stochastic heuristic. 
Table 5. Results for 100 instances on $10+10$ nodes with increasing density, 10 trials each

\begin{tabular}{|l|r|r|r|r|r|r|r|r|r|}
\hline$n_{i}$ & $m$ & Min & LR-Opt & Bary & Median & Stoch & Gre-ins & Gre-swi & Split \\
\hline 10 & 10 & 0.29 & 0.30 & 0.31 & 0.71 & 0.73 & 2.10 & 3.95 & 0.42 \\
& & 100.00 & 103.45 & 106.90 & 244.83 & 251.72 & 724.14 & 1362.07 & 144.83 \\
& & 1.10 & 0.11 & 0.08 & 0.08 & 0.27 & 0.21 & 0.16 & 0.38 \\
\hline 10 & 20 & 11.62 & 12.50 & 12.44 & 16.57 & 17.44 & 30.55 & 21.00 & 13.83 \\
& & 100.00 & 107.57 & 107.06 & 142.60 & 150.09 & 262.91 & 180.72 & 119.02 \\
& & 3.89 & 0.18 & 0.12 & 0.13 & 0.52 & 0.38 & 0.34 & 0.64 \\
\hline 10 & 30 & 56.60 & 57.27 & 57.46 & 68.66 & 66.33 & 97.22 & 62.59 & 58.30 \\
& & 100.00 & 101.18 & 101.52 & 121.31 & 117.19 & 171.77 & 110.58 & 103.00 \\
& & 14.06 & 0.26 & 0.17 & 0.15 & 0.68 & 0.60 & 0.62 & 1.01 \\
\hline 10 & 40 & 146.89 & 147.35 & 147.73 & 166.41 & 159.31 & 205.97 & 150.34 & 148.24 \\
& & 100.00 & 100.31 & 100.57 & 113.29 & 108.46 & 140.22 & 102.35 & 100.92 \\
& & 43.02 & 0.36 & 0.21 & 0.18 & 0.79 & 0.90 & 1.02 & 1.45 \\
\hline 10 & 50 & 276.78 & 277.11 & 277.78 & 304.62 & 292.34 & 363.43 & 277.85 & 277.61 \\
& & 100.00 & 100.12 & 100.36 & 110.06 & 105.62 & 131.31 & 100.39 & 100.30 \\
& & 91.58 & 0.47 & 0.26 & 0.22 & 0.87 & 1.23 & 1.50 & 2.03 \\
\hline 10 & 60 & 463.17 & 463.76 & 464.07 & 499.41 & 478.48 & 565.63 & 464.54 & 464.17 \\
& & 100.00 & 100.13 & 100.19 & 107.82 & 103.31 & 122.12 & 100.30 & 100.22 \\
& & 206.61 & 0.59 & 0.32 & 0.25 & 0.96 & 1.65 & 2.15 & 2.67 \\
\hline 10 & 70 & 698.35 & 698.75 & 699.23 & 745.00 & 712.78 & 816.80 & 699.37 & 699.04 \\
& & 100.00 & 100.06 & 100.13 & 106.68 & 102.07 & 116.96 & 100.15 & 100.10 \\
& & 379.12 & 0.68 & 0.34 & 0.29 & 1.03 & 2.23 & 2.78 & 3.30 \\
\hline 10 & 80 & 1008.38 & 1008.62 & 1008.88 & 1070.82 & 1018.66 & 1120.31 & 1008.96 & 1008.94 \\
& & 100.00 & 100.02 & 100.05 & 106.19 & 101.02 & 111.10 & 100.06 & 100.06 \\
& & 763.53 & 0.81 & 0.37 & 0.31 & 1.11 & 2.70 & 3.39 & 3.89 \\
\hline 10 & 90 & 1405.57 & 1406.14 & 1406.22 & 1490.03 & 1410.31 & 1461.52 & 1406.43 & 1406.44 \\
& & 100.00 & 100.04 & 100.05 & 106.01 & 100.34 & 103.98 & 100.06 & 100.06 \\
& & 1549.12 & 0.70 & 0.33 & 0.34 & 1.17 & 2.86 & 3.13 & 3.53 \\
\hline
\end{tabular}

Table 6. Results for 10 instances of sparse graphs

\begin{tabular}{|r|r|r|r|r|r|r|r|r|}
\hline$n_{i}$ & $m$ & LR-Opt & Bary & Median & Stoch & Gre-ins & Gre-swi & Split \\
\hline 10 & 20 & 19.70 & 15.70 & 25.70 & 27.20 & 35.80 & 34.20 & 20.90 \\
& & 0.02 & 0.02 & 0.01 & 0.05 & 0.04 & 0.04 & 0.06 \\
\hline 20 & 40 & 73.70 & 72.50 & 79.60 & 132.50 & 170.70 & 237.70 & 91.20 \\
& & 0.10 & 0.03 & 0.04 & 0.36 & 0.17 & 0.17 & 0.41 \\
\hline 30 & 60 & 176.00 & 147.90 & 188.50 & 288.20 & 442.30 & 549.80 & 208.30 \\
& & 0.48 & 0.10 & 0.09 & 1.18 & 0.49 & 0.48 & 1.33 \\
\hline 40 & 80 & 309.80 & 273.30 & 374.20 & 555.70 & 760.60 & 1207.00 & 368.80 \\
& & 1.81 & 0.17 & 0.14 & 2.72 & 0.93 & 0.67 & 3.45 \\
\hline 50 & 100 & 457.70 & 392.30 & 561.90 & 824.40 & 1284.40 & 1971.20 & 548.10 \\
& & 5.87 & 0.25 & 0.17 & 5.92 & 1.37 & 1.10 & 7.14 \\
\hline 60 & 120 & 645.60 & 567.00 & 811.20 & 1219.90 & 1854.80 & 2867.90 & 811.10 \\
& & 13.34 & 0.38 & 0.24 & 8.58 & 2.24 & 1.87 & 10.52 \\
\hline 70 & 140 & 861.30 & 764.60 & 1146.20 & 1689.30 & 2549.30 & 4122.80 & 1032.40 \\
& & 24.95 & 0.55 & 0.34 & 14.09 & 2.89 & 2.19 & 19.48 \\
\hline 80 & 160 & 1246.10 & 1080.70 & 1481.30 & 2183.30 & 3279.40 & 5495.90 & 1467.70 \\
& & 62.65 & 0.68 & 0.52 & 21.09 & 4.58 & 3.22 & 25.01 \\
\hline 90 & 180 & 1697.70 & 1272.40 & 1848.00 & 2859.50 & 4280.00 & 6853.70 & 1762.40 \\
& & 86.37 & 1.10 & 0.57 & 31.84 & 6.41 & 4.30 & 38.36 \\
\hline 100 & 200 & 2027.30 & 1555.10 & 2084.10 & 3453.10 & 5405.00 & 8796.30 & 2208.40 \\
& & 178.93 & 1.46 & 0.82 & 40.23 & 7.41 & 5.25 & 47.78 \\
\hline
\end{tabular}


Table 7. Results for 10 instances of sparse graphs, 10 trials each

\begin{tabular}{|r|r|r|r|r|r|r|r|r|}
\hline$n_{i}$ & $m$ & LR-Opt & Bary & Median & Stoch & Gre-ins & Gre-swi & Split \\
\hline 10 & 20 & 13.60 & 12.70 & 18.70 & 17.50 & 30.00 & 22.30 & 14.70 \\
& & 0.12 & 0.15 & 0.12 & 0.55 & 0.40 & 0.34 & 0.68 \\
\hline 20 & 40 & 51.00 & 48.30 & 59.10 & 89.00 & 150.80 & 163.40 & 63.70 \\
& & 0.98 & 0.42 & 0.39 & 3.61 & 1.82 & 1.58 & 3.93 \\
\hline 30 & 60 & 133.40 & 117.00 & 145.80 & 228.60 & 421.30 & 422.10 & 160.10 \\
& & 5.55 & 0.96 & 0.76 & 11.48 & 4.59 & 4.18 & 13.13 \\
\hline 40 & 80 & 234.10 & 212.40 & 271.40 & 432.80 & 724.50 & 949.80 & 279.90 \\
& & 18.45 & 1.75 & 1.29 & 26.57 & 8.25 & 7.42 & 31.26 \\
\hline 50 & 100 & 384.20 & 325.60 & 407.30 & 715.60 & 1245.60 & 1715.90 & 462.70 \\
& & 52.01 & 2.79 & 2.06 & 51.33 & 13.59 & 11.60 & 60.80 \\
\hline 60 & 120 & 541.10 & 479.90 & 599.90 & 1106.80 & 1909.70 & 2472.10 & 654.00 \\
& & 128.12 & 4.38 & 2.93 & 92.08 & 21.97 & 18.27 & 114.07 \\
\hline 70 & 140 & 733.20 & 641.30 & 858.00 & 1489.30 & 2514.30 & 3640.00 & 896.90 \\
& & 304.08 & 5.79 & 3.82 & 139.95 & 30.18 & 23.22 & 175.83 \\
\hline 80 & 160 & 1022.90 & 903.70 & 1145.10 & 1993.30 & 3248.70 & 4843.50 & 1169.60 \\
& & 619.36 & 7.57 & 5.28 & 204.64 & 38.96 & 31.18 & 264.82 \\
\hline 90 & 180 & 1282.50 & 1044.70 & 1323.70 & 2516.50 & 4209.10 & 6228.20 & 1466.40 \\
& & 1134.67 & 10.81 & 6.55 & 307.44 & 57.13 & 43.19 & 377.72 \\
\hline 100 & 200 & 1599.20 & 1313.20 & 1793.20 & 3119.40 & 5323.90 & 8145.30 & 1807.60 \\
& & 2313.48 & 13.76 & 8.02 & 402.74 & 67.13 & 50.24 & 504.25 \\
\hline
\end{tabular}

\section{References}

[CPLEX] CPLEX: Using the CPLEX callable library and the CPLEX mixed integer library. CPLEX Optimization Inc. (1993)

[D94] Dresbach, S.: A New Heuristic Layout Algorithm for DAGs. Derigs, Bachem \& Drexl (eds.) Operations Research Proceedings 1994, Springer Verlag, Berlin (1994) 121-126

[D95] Dresbach, S.: Personal communication. (1995)

[EK 86] Eades, P., and D. Kelly: Heuristics for Reducing Crossings in 2-Layered Networks. Ars Combinatoria 21-A (1986) 89-98

[EW94] Eades, P., and N.C. Wormald: Edge crossings in Drawings of Bipartite Graphs. Algorithmica 10 (1994) 379-403

[GJ83] Garey, M.R., and D.S. Johnson: Crossing Number is NP-Complete. SIAM J. on Algebraic and Discrete Methods 4 (1983) 312-316

[GJR84a] Grötschel, M., M. Jünger, and G. Reinelt: A cutting plane algorithm for the linear ordering problem. Operations Research 32 (1984) 1195-1220

[GJR84b] Grötschel, M., M. Jünger, and G. Reinelt: Optimal triangulation of large real world input-output matrices. Statistische Hefte 25 (1984) 261-295

[GJR85] Grötschel, M., M. Jünger, and G. Reinelt: Facets of the linear ordering polytope. Mathematical Programming 33 (1985) 43-60

[K93] Knuth, D.E.: The Stanford GraphBase: A Platform for Combinatorial Computing. ACM Press, Addison-Wesley Publishing Company (1993) New York

[STT81] Sugiyama, K., S. Tagawa, and M. Toda: Methods for Visual Understanding of Hierarchical System Structures. IEEE Trans. Syst. Man, Cybern., SMC11 (1981) 109-125

[W77] Warfield, J.N.: Crossing Theory and Hierarchy Mapping. IEEE Trans. Syst. Man, Cybern., SMC-7 (1977) 505-523 